\title{
Assistência Farmacêutica na Saúde Mental: um diagnóstico dos Centros de Atenção Psicossocial
}

\author{
Pharmaceutical Assistance in Mental Health: \\ a diagnosis of Psychosocial Care Centers
}

Sarah Nascimento Silva ${ }^{1}$ Marina Guimarães Lima ${ }^{1}$
${ }^{1}$ Programa de PósGraduação em Medicamentos e Assistência Farmacêutica, Departamento de Farmácia Social, Universidade Federal de Minas Gerais. Av. Presidente Antônio Carlos 6627, Pampulha. 31270-901 Belo Horizonte MG Brasil. sarahnsilva@gmail.com

\begin{abstract}
The study evaluated the conditions of Pharmaceutical Assistance (PA) in Psychosocial Care Centers (CAPS) through a cross-sectional study in 15 CAPS located in the Médio Paraopeba region of the State of Minas Gerais. Data collection was conducted through direct observation of CAPS and interviews with pharmacists and managers of PA. The instruments were based on indicators proposed by the WHO, technical documents for the organization of $P A$, from legislation and recommendations seeking pharmaceutical Best Practices. There were 13 dispensing units, nine of them within the CAPS. The services presented updated lists of essential medicines with high availability of the main drugs used in mental health treatment. All of the cities participated in an inter-municipal consortium for the purchase of medicines. The complete identification of drugs was present in only seven services and all had failings in traceability of medicines dispensed. In the CAPS there is only one pharmacist during part of the operating peri$o d$. The need for greater participation of the pharmacist was observed in the control and standardization of the activities of CAPS and especially in care activities comprising the benchmark team in Mental Health.
\end{abstract}

Key words Pharmaceutical services, Mental health, Evaluation of health services
Resumo O estudo avaliou as condições da Assistência Farmacêutica (AF) nos Centros de Atenção Psicossocial (CAPS) por meio de um estudo transversal em 15 CAPS na região do Médio Paraopeba, Minas Gerais. A coleta de dados foi realizada por meio de observação direta dos CAPS e entrevistas com farmacêuticos e gestores da AF. Os instrumentos foram baseados em indicadores propostos pela OMS, documentos técnicos para organização da $A F$, na legislação e recomendações que visam as Boas Práticas Farmacêuticas. Foram identificadas 13 unidades de dispensação, sendo nove delas internas aos CAPS. Os serviços apresentaram listas de medicamentos essenciais atualizadas e alta disponibilidade dos principais utilizados nos tratamentos da saúde mental. Todas as cidades participavam de um consórcio intermunicipal para aquisição de medicamentos. A identificação completa dos medicamentos estava presente em apenas sete serviços e todos apresentaram falhas na rastreabilidade dos medicamentos que foram dispensados. Nos CAPS há apenas um profissional farmacêutico em parte do período de funcionamento e foi identificada apenas uma atividade direcionada aos usuários relacionada ao uso de medicamentos em um dos CAPS. Verificou-se a necessidade de maior participação do farmacêutico no controle e padronização das atividades dos CAPS $e$ principalmente nas atividades assistenciais compondo a equipe de referência em Saúde Mental.

Palavras-chave Assistência farmacêutica, Saúde mental, Avaliação de serviços de saúde 


\section{Introdução}

Os transtornos mentais contribuem significativamente para a carga global de doenças da população e estão associados a deficiências, dificuldades no diagnóstico de outras doenças, além de interferir em custos para a saúde, diminuição da produtividade e qualidade de vida ${ }^{1,2}$. Os transtornos decorrentes da dependência química e o abuso de drogas apresentaram crescimento substancial nos últimos anos e também compreendem um importante desafio para os sistemas de saúde em países em desenvolvimento ${ }^{3}$. Um estudo multicêntrico, realizado recentemente em São Paulo, Brasil, apontou elevadas estimativas de prevalência de transtornos mentais em relação a outras cidades em todo o mundo ${ }^{4}$. Transtornos mentais como a ansiedade e a depressão são comuns em muitos usuários da atenção primária das principais capitais do país, as altas taxas estão associadas às mulheres, aos desempregados, pessoas com baixa escolaridade e baixa renda ${ }^{5}$.

Os Centros de Atenção Psicossocial (CAPS) foram criados como serviços regionalizados, integrando a rede de atenção à Saúde Mental e proporcionando o atendimento de particularidades locais. Os CAPS são integrantes de uma nova modalidade de atenção implantada no Sistema Único de Saúde (SUS) que envolve a construção de uma política pública baseada em práticas multiprofissionais e interdisciplinares ${ }^{6,7}$. Considerados dispositivos estratégicos na desinstitucionalização de portadores de transtornos mentais, os CAPS estão fundamentados na noção de apoio matricial e possuem políticas públicas convergentes com a Atenção Básica ${ }^{8}$. Ao longo dos últimos anos, o número de CAPS cresceu significativamente, acompanhado da redução de leitos hospitalares, por meio de um processo planejado e articulado em consonância com a expansão da rede de ações e serviços territoriais?.

Os CAPS, como instituições de referência para a Saúde Mental no tratamento e atendimento de crises e urgências, têm o uso de medicamentos como uma atividade comum e inerente ao plano terapêutico ${ }^{10,11}$. O uso de medicamentos nestes centros requer a organização própria do serviço de Assistência Farmacêutica (AF), uma vez que esta contempla diversas atividades voltadas não só para sua disponibilidade, mas também para a conservação, o controle de qualidadee atividades assistenciais a fim de garantir a segurança e a efetividade dos medicamentos. São necessários ainda mecanismos de acompanhamento e avaliação da utilização de medicamentos, difusão de informações, além da educação permanente dos profissionais de saúde, assegurando seu uso racional $^{12,13}$.

A AF nos CAPS demanda adequado planejamento a fim de cumprir com seu objetivo de proporcionar a assistência terapêutica integral, promoção e recuperação da saúde, podendo as farmácias destes centros se tornarem referência na dispensação de medicamentos da saúde mental ${ }^{14}$. Em um modelo de atenção colaborativa a participação do farmacêutico nos CAPS é fundamental, visto que este profissional pode estabelecer o primeiro contato com o usuário ou atuar como um elo com os demais profissionais da equipe de saúde mental, principalmente com o médico, e participar em estratégias de adesão atuando no cuidado à saúde mental ${ }^{15,16}$.

A avaliação dos serviços de saúde faz parte do processo de planejamento e gestão, sendo capaz de subsidiar parâmetros importantes no desenvolvimento de novas estratégias e alterações no serviço. Os estudos de avaliação dos serviços de Saúde Mental ainda são escassos ${ }^{17}$ e os mesmos não avaliam critérios específicos das condições da AF nestas unidades, serviço considerado indissociável no modelo de assistência atualmente praticado no país ${ }^{13,18}$. A fim de preencher esta lacuna e conhecer melhor alguns aspectos, condições e instalações nos serviços de saúde mental este estudo teve o objetivo de descrever as condições da AF nos CAPS na região denominada Médio Paraopeba no Estado de Minas Gerais.

\section{Métodos}

Foi realizado um estudo transversal, quantitativo sobre aspectos da organização da Assistência Farmacêutica nos CAPS na região do Médio Paraopeba, Minas Gerais. Os municípios elegíveis para o estudo foram aqueles que pertenciam à região, ofertavam o serviço CAPS e cujos gestores das áreas de Saúde Mental e AF concordaram em participar da pesquisa.

Os instrumentos de coleta de dados foram delineados para descrever as condições da AF em suas atividades constitutivas de seleção, programação, aquisição, armazenamento e dispensação de medicamentos psicotrópicos. Foram desenvolvidos dois formulários semiestruturados: um destinado à observação direta dos CAPS pelo pesquisador e outro para coleta de dados sobre os serviços por meio de entrevista dirigida com os farmacêuticos atuantes nos CAPS e os coordenadores da AF em cada município. Os gestores foram entrevistados quanto aos aspectos da aquisição e seleção de medicamentos nos municípios, 
em função destas etapas serem executadas para o atendimento de todos os serviços de saúde e não apenas os CAPS. Perguntas sobre as demais etapas de organização da AF, destinadas a particularidades de cada unidade de dispensação, foram direcionadas aos farmacêuticos alocados no serviço e aos gestores da AF na falta deste profissional.

Como referência para o desenvolvimento dos instrumentos de coleta de dados foram empregados alguns indicadores propostos pela Organização Mundial de Saúde (OMS) para a avaliação do acesso, qualidade e uso racional de medicamentos em inquéritos domiciliares e em serviços de saúde, denominados de indicadores de nível $\mathrm{II}^{19}$. Os indicadores de acesso propostos pela OMS contemplam a capacidade aquisitiva e a disponibilidade de medicamentos; os de qualidade verificam a preservação da integridade dos produtos e a qualidade de alguns processos de trabalho; e os de uso racional contemplam questões ligadas à prescrição e a aspectos organizacionais. Dentre os diferentes indicadores da OMS, selecionaram-se aqueles diretamente relacionados com as atividades constitutivas da AF em serviços de Saúde Mental. Foram inclusos também diversos critérios extraídos de instruções técnicas para organização da $\mathrm{AF}^{12,20,21}$ que apresentam requisitos básicos para o funcionamento do ciclo da AF, a legislação que regulamenta a dispensação de medicamentos psicotrópicos no Brasil ${ }^{22}$ e outras recomendações especificas que visam as Boas Práticas Farmacêuticas ${ }^{23}$. Os critérios disponíveis nestes documentos foram agrupados e combinados de maneira a caracterizar cada uma das etapas do ciclo da AF (Quadro 1).

A variável "disponibilidade de medicamentos" utilizou o conceito de medicamento essencial estabelecido pela OMS, para o qual são eleitos 15 medicamentos para o tratamento das principais condições de saúde da população. Por se tratar de um estudo restrito aos CAPS, a elaboração desta lista usou como ponto de partida os medicamentos destinados aos tratamentos da Saúde Mental presentes na Relação Nacional de Medicamentos (RENAME) 201324. Este elenco incluiu todos os medicamentos da saúde mental presentes no Componente Básico da Assistência Farmacêutica em pelo menos uma apresentação farmacêutica.

A descrição da etapa de dispensação de medicamentos verificou critérios de adequação propostos pela OMS para esta área, similar a uma lista de verificação, e também itens sobre a atuação dos farmacêuticos nos CAPS. Os farmacêuticos foram questionados em entrevista sobre a intera- ção com a equipe multidisciplinar, participação no plano de cuidado dos usuários, realização de orientações básicas aos usuários e atividades específicas conduzidas por estes profissionais nos CAPS. Foram investigados também a presença de Protocolos e Diretrizes que delimitem condutas específicas para o uso de medicamentos nos CAPS, notificação de reações adversas pelas farmácias e relatos de erros de medicação, uso de dose acima da recomendada ou erro de dispensação nos serviços por meio de entrevista com os gestores dos CAPS e farmacêuticos atuantes nos serviços.

Foi realizado um teste piloto em um dos municípios participantes para avaliar a adequação dos formulários e a qualidade da coleta de dados. A coleta de dados foi realizada no período de abril a junho de 2014 por um pesquisador graduado e previamente treinado que observou o local em pelo menos um dia de funcionamento da instituição. Foi realizada dupla digitação dos dados coletados dos questionários no software Microsoft Excel 2010.

A análise de dados foi realizada por estatística descritiva. Foi descrita a frequência das variáveis observadas em cada etapa da AF pelo pesquisador e aquelas coletadas durante as entrevistas dirigidas com os farmacêuticos e gestores dos serviços. Medidas de tendência central (média e mediana) e medidas de variação (desvio padrão) foram empregadas para análise das variáveis quantitativas. A unidade de análise para as etapas de seleção e aquisição foi o município e para as demais foram as unidades de dispensação que atendiam a demanda dos CAPS. Para análise dos dados foram utilizados os softwares Microsoft Excel 2010 e Statistical Package for the Social Sciences (SPSS), versão 19.0.

O estudo seguiu as recomendações éticas constantes na resolução do Conselho Nacional de Saúde CNS 466/2012 e foi aprovado pelo Comitê de Ética em Pesquisa da Universidade Federal de Minas Gerais.

\section{Resultados}

O estudo foi realizado em sete cidades cujas populações somavam 1.359.413 habitantes em $2014^{25}$. Foram inclusos todos os 15 CAPS presentes nas cidades de Betim (MG), Brumadinho (MG), Contagem (MG), Esmeraldas (MG), Ibirité (MG), Igarapé (MG) e São Joaquim de Bicas (MG). Foram detectadas 13 unidades de dispensação, dentre farmácias ou dispensários de medicamentos, para o atendimento dos usuários dos 
Quadro 1. Critérios utilizados no diagnóstico da Assistência farmacêutica, documentos norteadores e estratégia de coleta dos dados, Região do Médio Paraopeba-MG, Brasil, 2014.

\begin{tabular}{|c|c|c|c|c|c|}
\hline $\begin{array}{l}\text { Etapas do ciclo da Assistência } \\
\text { Farmacêutica }\end{array}$ & $\begin{array}{l}\text { Critérios } \\
\text { adaptados } \\
\text { de WHO }\end{array}$ & $\begin{array}{l}\text { Documentos } \\
\text { Técnicos }\end{array}$ & $\begin{array}{c}\text { Legislação } \\
\text { vigente }\end{array}$ & Outros & $\begin{array}{l}\text { Estratégia de } \\
\text { coleta e fonte } \\
\text { de dado }\end{array}$ \\
\hline \multicolumn{6}{|l|}{ Seleção } \\
\hline $\begin{array}{l}\text { Disponibilidade de Lista de } \\
\text { Medicamentos Essenciais no município } \\
\text { (REMUME) e divulgação para o CAPS }\end{array}$ & $\mathrm{x}$ & $\mathrm{x}$ & & & $\begin{array}{l}\text { Entr. Gestor AF/ } \\
\text { Obs.direta }\end{array}$ \\
\hline $\begin{array}{l}\text { REMUME atualizada (até } 2 \text { anos - última } \\
\text { revisão) }\end{array}$ & & $\mathrm{x}$ & & & $\begin{array}{l}\text { Entr. Gestor AF/ } \\
\text { Obs.direta }\end{array}$ \\
\hline $\begin{array}{l}\text { Presença de Comissão de Farmácia } \\
\text { e Terapêutica (CFT) e profissionais } \\
\text { envolvidos }\end{array}$ & & $\mathrm{x}$ & & & Entr. Gestor AF \\
\hline $\begin{array}{l}\text { Revisões e atualizações da Lista de } \\
\text { Medicamentos Essenciais envolvem } \\
\text { profissionais do CAPS }\end{array}$ & & & & $\mathrm{x}$ & Entr. Gestor AF \\
\hline \multicolumn{6}{|l|}{ Aquisição } \\
\hline $\begin{array}{l}\text { Licitação/Registro de preço vigente para } \\
\text { aquisição de medicamentos principais* }\end{array}$ & & $\mathrm{x}$ & & & Entr. Gestor AF \\
\hline $\begin{array}{l}\text { Cadastro de fornecedores e controle } \\
\text { de pedidos (quantidades empenhadas } \\
\text { entregues de cada processo licitatório) }\end{array}$ & & $\mathrm{x}$ & & & Entr. Gestor AF \\
\hline $\begin{array}{l}\text { Existência de requisitos técnicos em } \\
\text { editais de licitação }\end{array}$ & & $\mathrm{x}$ & & & Entr. Gestor AF \\
\hline $\begin{array}{l}\text { Participação em consórcio para aquisição } \\
\text { de medicamentos/insumos }\end{array}$ & & & & $\mathrm{x}$ & Entr. Gestor AF \\
\hline \multicolumn{6}{|l|}{ Programação } \\
\hline $\begin{array}{l}\text { Profissional responsável pela } \\
\text { programação da AF na farmácia e que } \\
\text { compreende conceitos sobre gerência } \\
\text { de estoques (consumo médio, estoque } \\
\text { mínimo, FEFO) }\end{array}$ & & & & & $\begin{array}{l}\text { Entr. Gestor AF/ } \\
\text { Obs.direta }\end{array}$ \\
\hline $\begin{array}{l}\text { Utilização de algum método de } \\
\text { programação (consumo histórico, perfil } \\
\text { populacional, oferta dos serviços) }\end{array}$ & & $\mathrm{x}$ & & & Entr. Gestor AF \\
\hline $\begin{array}{l}\text { Realização de conferência dos } \\
\text { medicamentos }\end{array}$ & & $\mathrm{x}$ & & & Entr. Gestor AF \\
\hline Realização de inventário freqüente & & $\mathrm{x}$ & & & Entr. Gestor AF \\
\hline $\begin{array}{l}\text { Existência de método de Controle de } \\
\text { estoque/ registros de abastecimento e } \\
\text { posição de estoque }\end{array}$ & $\mathrm{x}$ & $\mathrm{x}$ & & & $\begin{array}{l}\text { Entr. Gestor AF/ } \\
\text { Obs.direta }\end{array}$ \\
\hline $\begin{array}{l}\text { Dados sobre disponibilidade contínua } \\
\text { dos medicamentos /tempo médio de } \\
\text { desabastecimentos }\end{array}$ & $\mathrm{x}$ & & & & $\begin{array}{l}\text { Entr. Gestor AF/ } \\
\text { Obs.direta }\end{array}$ \\
\hline $\begin{array}{l}\text { Manutenção de registros de } \\
\text { movimentação de estoques }\end{array}$ & $\mathrm{x}$ & $\mathrm{x}$ & & & $\begin{array}{l}\text { Entr. Gestor AF/ } \\
\text { Obs.direta }\end{array}$ \\
\hline \multicolumn{6}{|l|}{ Armazenamento } \\
\hline Local amplo, limpo e arejado & $\mathrm{x}$ & $\mathrm{x}$ & & & Obs.direta \\
\hline $\begin{array}{l}\text { Ambiente com métodos de controle de } \\
\text { temperatura protegido da ação direta da } \\
\text { luz solar }\end{array}$ & $\mathrm{x}$ & $\mathrm{x}$ & & & Obs.direta \\
\hline Área para quarentena & & $\mathrm{x}$ & & & Obs.direta \\
\hline $\begin{array}{l}\text { Identificação das prateleiras/ } \\
\text { armazenamento de forma sistemática }\end{array}$ & $\mathrm{x}$ & $\mathrm{x}$ & & & Obs.direta \\
\hline
\end{tabular}


Quadro 1. continuação

\begin{tabular}{|c|c|c|c|c|c|}
\hline $\begin{array}{c}\text { Etapas do ciclo da Assistência } \\
\text { Farmacêutica }\end{array}$ & $\begin{array}{l}\text { Critérios } \\
\text { adaptados } \\
\text { de WHO }\end{array}$ & $\begin{array}{l}\text { Documentos } \\
\text { Técnicos }\end{array}$ & $\begin{array}{l}\text { Legislação } \\
\text { vigente }\end{array}$ & Outros & $\begin{array}{l}\text { Estratégia de } \\
\text { coleta e fonte } \\
\text { de dado }\end{array}$ \\
\hline \multicolumn{6}{|l|}{ Dispensação } \\
\hline $\begin{array}{l}\text { Disponibilidade dos medicamentos } \\
\text { principais* }\end{array}$ & $\mathrm{x}$ & & & & Obs.direta \\
\hline $\begin{array}{l}\text { Local de dispensação adequado } \\
\text { (conforme critérios da lista de verificação } \\
\text { OMS) }\end{array}$ & $\mathrm{x}$ & & & & Obs.direta \\
\hline Cadastro de usuários & & & & & Obs.direta \\
\hline Dispensação mediante prescrição médica & & $\mathrm{x}$ & $\mathrm{x}$ & & Obs.direta \\
\hline $\begin{array}{l}\text { Informação de lote e validade dos } \\
\text { medicamentos recebidos }\end{array}$ & & $\mathrm{x}$ & $\mathrm{x}$ & & $\begin{array}{l}\text { Entr. Farm./ } \\
\text { Obs.direta } \\
\end{array}$ \\
\hline $\begin{array}{l}\text { Informação de lote e validade dos } \\
\text { medicamentos dispensados }\end{array}$ & & $\mathrm{x}$ & $\mathrm{x}$ & & $\begin{array}{l}\text { Entr. Farm./ } \\
\text { Obs.direta }\end{array}$ \\
\hline Escrituração das prescrições & & $\mathrm{x}$ & & & Obs.direta \\
\hline Medicamentos sob controle (trancados) & & $\mathrm{x}$ & & & Obs. direta \\
\hline Manutenção de registros de dispensação & $\mathrm{x}$ & $\mathrm{x}$ & $\mathrm{x}$ & & Obs. direta \\
\hline $\begin{array}{l}\text { Identificação completa dos } \\
\text { medicamentos dispensados e } \\
\text { administrados/fracionamento de acordo } \\
\text { com critérios da legislação }\end{array}$ & $\mathrm{x}$ & & $\mathrm{x}$ & & Obs. direta \\
\hline $\begin{array}{l}\text { Presença do farmacêutico responsável no } \\
\text { dispensário de medicamentos da saúde } \\
\text { mental (CAPS ou farmácia central) e } \\
\text { carga horária no estabelecimento }\end{array}$ & $\mathrm{x}$ & $\mathrm{x}$ & & & $\begin{array}{l}\text { Entr. Gestor AF/ } \\
\text { Obs.direta }\end{array}$ \\
\hline $\begin{array}{l}\text { Orientação do usuário durante a } \\
\text { dispensação de medicamentos }\end{array}$ & & $\mathrm{x}$ & & $\mathrm{x}$ & Entr. Farm. \\
\hline
\end{tabular}

Entr. Gestor AF: Entrevista gestor da Assistência Farmacêutica; Obs. Diret: Observação direta; Entr. Farm: Entrevista

Farmacêutico. ${ }^{*}$ lista de medicamentos elaboradas para o estudo.

CAPS avaliados, sendo nove delas localizadas no interior dos CAPS e quatro em farmácias centrais responsáveis pelo fornecimento dos medicamentos a toda população do município, inclusive aos usuários da saúde mental assistidos pelos CAPS de menor porte.

A organização da etapa de seleção de medicamentos estava presente em todas as cidades inclusas no estudo e documentada por meio da Relação Municipal de Medicamentos Essenciais (REMUME) atualizada, ou seja, elaborada nos últimos dois anos. Este documento estava presente em todas as 13 unidades de dispensação e em 14 CAPS avaliados. A participação de profissionais dos CAPS na elaboração ou revisão desta relação de medicamentos foi declarada em quatro cidades e a atuação de Comissão de Farmácia e Terapêutica (CFT) estava presente em apenas duas cidades.

Em relação à aquisição de medicamentos, em todas as cidades foi declarada a existência de registro de preço/licitação vigente para diversos medicamentos da saúde mental, inclusive para todos os 15 da lista de medicamentos elaborada para este estudo. Os gestores informaram ainda sobre a existência de cadastro de fornecedores, controle de pedidos e a existência de requisitos técnicos nos editais de aquisição dos medicamentos. Em todos os municípios a participação no Consórcio Intermunicipal de Saúde do Médio Paraopeba (MG) (CISMEP) foi confirmada, seja por meio de adesão do município a diversos serviços oferecidos pelo consórcio ou a atas para a aquisição de medicamentos.

A programação de cada uma destas unidades de dispensação era realizada por um funcionário designado ou o próprio farmacêutico, ambos declararam compreender conceitos básicos relacionados à gerência de estoques. Em 12 dispensários os responsáveis pela atividade declararam aplicar os conceitos e algum dos métodos de cálculo recomendados para a programação da 
AF. Os farmacêuticos realizavam controle de estoque, conferência dos medicamentos recebidos e inventários frequentes por meio de contagem mensal (Tabela 1).

Foi verificada a existência de controle de estoque em 12 unidades de dispensação e em quatro delas registros e informações precisas acerca dos históricos de desabastecimentos que ocorreram ao longo do último ano para cada medicamento avaliado. Estes dispensários com registro de histórico possuíam controle de estoque realizado por fichas de prateleira, ou livro de escrituração de medicamentos movimentados. Os demais serviços continham anotações, listas de produtos recebidos e mapas sem detalhes sobre o consumo de cada medicamento, período exato de desabastecimentos e outras estimativas que pudessem avaliar a disponibilidade contínua.
As condições de armazenamento em cada uma das unidades de dispensação foi avaliada, 12 delas apresentaram instalações físicas adequadas, ou seja, apresentaram local amplo, limpo e arejado para o armazenamento dos medicamentos, com espaço separado para quarentena dos impróprios para o uso. O controle de temperatura era realizado em quatro unidades de dispensação e em todas a identificação das prateleiras destinadas ao armazenamento dos medicamentos estava devidamente sinalizada (Tabela 1).

As unidades de dispensação avaliadas apresentaram local adequado para a dispensação dos medicamentos, ao cumprirem mais de $2 / 3$ dos requisitos da lista de verificação da área de dispensação (Tabela 2). Em todas as áreas avaliadas não havia indícios de animais ou insetos no local, além de apresentarem janelas ou saídas de

Tabela 1. Descrição das unidades dispensadoras de medicamentos quanto às etapas de programação, armazenamento e dispensação da Saúde Mental, Região do Médio Paraopeba-MG, Brasil, 2014.

\begin{tabular}{|c|c|}
\hline Critério observado & Frequência absoluta $(\mathrm{N})$ \\
\hline \multicolumn{2}{|l|}{ Programação } \\
\hline Funcionário designado responsável pela programação & 13,0 \\
\hline Aplicação dos conceitos e cálculos para a realização da programação & 12,0 \\
\hline Método de programação gerou desabastecimento & 5,0 \\
\hline \multicolumn{2}{|l|}{ Armazenamento } \\
\hline Local adequado (amplo, limpo e arejado) & 12,0 \\
\hline Limpeza adequada & 12,0 \\
\hline Realização de conferência dos medicamentos & 12,0 \\
\hline Controle de estoque & 12,0 \\
\hline Realização de inventário frequente & 12,0 \\
\hline Aferição da temperatura & 4,0 \\
\hline Área para quarentena & 12,0 \\
\hline Identificação das prateleiras & 13,0 \\
\hline \multicolumn{2}{|l|}{ Dispensação } \\
\hline Local de dispensação adequado (conforme critérios da lista de verificação OMS) & 13,0 \\
\hline Registro das dispensações & 13,0 \\
\hline Orientação do usuário durante a dispensação & 11,0 \\
\hline Farmacêutico responsável CAPS ou farmácia central & 13,0 \\
\hline Medicamentos sob controle (trancados) & 12,0 \\
\hline Escrituração das prescrições & 2,0 \\
\hline Informação de lote e validade dos medicamentos recebidos & 12,0 \\
\hline Informação de lote e validade dos medicamentos dispensados & 0,0 \\
\hline Cadastro de usuários & 11,0 \\
\hline Notificação de reação adversa & 2,0 \\
\hline
\end{tabular}


ar. Os medicamentos principais da Saúde Mental, listados para este estudo, estavam disponíveis em grande parte dos dispensários (Tabela 3). A disponibilidade média dos medicamentos principais da saúde mental foi de 11,9 para uma lista de 15 farmacos.

Em todos os dispensários havia um farmacêutico responsável pelo serviço, sendo que em 11 unidades de dispensação havia registro/cadas- tro de usuários e os farmacêuticos relataram que os usuários são orientados durante a dispensação dos medicamentos. Em todos os serviços as dispensações são realizadas mediante apresentação da prescrição médica.

Quanto à rastreabilidade das informações nas unidades de dispensação, 12 delas possuem informações acerca dos lotes e validade dos medicamentos recebidos. Todas as unidades dispen-

Tabela 2. Condições do local de dispensação de medicamentos da Saúde Mental, Região do Médio Paraopeba-MG, Brasil, 2014.

\begin{tabular}{lc}
\hline \multicolumn{1}{c}{ Condição Avaliada } & $\begin{array}{c}\text { Frequência } \\
\text { absoluta }(\mathbf{N})\end{array}$ \\
\hline Existência de métodos para controle da temperatura & 12,0 \\
Existência de janelas ou saídas de ar & 13,0 \\
Proteção direta quanto à luz solar & 12,0 \\
Área isenta de umidade & 12,0 \\
Medicamentos não são armazenados diretamente no chão & 12,0 \\
Medicamentos armazenados de forma sistemática & 12,0 \\
Medicamentos armazenados de acordo com prazo de validade & 12,0 \\
Não há evidências de insetos e animais no local & 13,0 \\
Comprimidos/cápsulas não são manipulados com as mãos nuas & 11,0 \\
\hline
\end{tabular}

Tabela 3. Disponibilidade de Medicamentos para o tratamento de transtornos mentais nas unidades de dispensação dos CAPS, Região do Médio Paraopeba-MG, Brasil, 2014.

\begin{tabular}{lcc}
\hline \multicolumn{1}{c}{ Medicamentos Saúde Mental } & $\begin{array}{c}\text { Unidades com estoque do } \\
\text { medicamento (N) }\end{array}$ & $\begin{array}{c}\text { Frequência } \\
\text { relativa }(\%)^{\mathrm{a}}\end{array}$ \\
\hline Carbamazepina 200mg comprimido & 12,0 & 92,3 \\
Carbonato de Lítio 300mg comprimido & 13,0 & 100,0 \\
Clonazepam 2mg comprimido & 12,0 & 92,3 \\
Cloridrato de Amitriptilina 25mg comprimido & 13,0 & 100,0 \\
Cloridrato de Biperideno 2mg comprimido & 13,0 & 100,0 \\
Cloridrato de Clomipramina 25mg comprimido & 13,0 & 100,0 \\
Cloridrato de Clorpromazina 100mg comprimido & 13,0 & 100,0 \\
Cloridrato de Fluoxetina 20mg comprimido & 13,0 & 100,0 \\
Cloridrato de Nortriptilina 50mg capsula & 4,0 & 30,8 \\
Diazepam 10mg & 12,0 & 92,3 \\
Fenitoína Sódica 100mg comprimido & 13,0 & 100,0 \\
Fenobarbital 100mg comprimido & 10,0 & 76,9 \\
Haloperidol 5mg comprimido & 13,0 & 100,0 \\
Valproato de Sódio/ Ácido Valpróico 500mg comprimido & 13,0 & 100,0 \\
Valproato de Sódio/Ácido Valpróico solução oral ou xarope & 11,0 & 84,6 \\
Disponibilidade Média & 11,9 & 91,3 \\
\hline
\end{tabular}

${ }^{a} \mathrm{O}$ percentual de disponibilidade foi obtido a partir do número de unidades de dispensação com o medicamento em estoque. 
sadoras realizam os registros das quantidades dispensadas, mas nenhuma apresentou registros e informações sobre lote e validade dos medicamentos dispensados ao usuário. A escrituração das prescrições dos medicamentos controlados pela Portaria SVS/MS no 344/1998, por meio de livro manual, era realizada em dois serviços e os demais não apresentaram nenhuma outra forma de escrituração das prescrições (Tabela 1).

Foram verificados outros aspectos como a identificação adequada e completa de comprimidos e ampolas dispensados por estes estabelecimentos e a realização de fracionamento. Ao realizar a observação direta da atividade de fracionamento nos CAPS, observou-se algumas condições precárias como bancadas de trabalho sem identificação, manipulação de cápsulas e comprimidos com mãos nuas pelos funcionários não sendo preservada a identificação dos medicamentos e outros requisitos exigidos para o fracionamento estabelecidos pela legislação vigente. Os comprimidos individualizados ou separados da embalagem primária contendo identificação básica, como nome, dosagem do medicamento e informações sobre lote e validade estavam presentes em apenas sete unidades de dispensação visitadas.

Dentre os 15 CAPS avaliados, nove deles possuem a presença de um farmacêutico no serviço cumprindo uma carga horária semanal que varia de 20 a 30 horas. Todos estes farmacêuticos atuantes nos CAPS declararam realizar interações com a equipe multidisciplinar na discussão de casos clínicos e realização de intervenções quando necessário. Dentre os farmacêuticos alocados nos CAPS, sete declararam participar do plano de cuidado dos usuários que demandavam maior atenção por toda a equipe multiprofissional. A realização de orientações básicas a uma pequena parte dos usuários incluía a liberação de medicamentos com sinalização dos horários de administração, indicação dos nomes e dosagens dos medicamentos em uso e rápida conversa com o usuário sobre a existência de dúvidas e sua adesão ao tratamento. Em apenas um CAPS foi relatada a realização de uma oficina com os usuários sobre o tema medicamentos, visando à orientação sobre o uso correto e racional dos da Saúde Mental. Ressalta-se, porém, que as atividades e intervenções farmacêuticas não geravam anotação em prontuário ou qualquer tipo de documentação.

Os gestores da AF declararam a existência de algumas ocorrências relacionadas aos erros de medicação, uso de dose acima da recomendada ou erro de dispensação em nove serviços. Todos os gestores e farmacêuticos entrevistados relata- ram que o uso de Protocolos Clínicos, recomendações ou Diretrizes Terapêuticas sobre o uso de medicamentos ou condutas específicas para o tratamento da Saúde Mental ou uso de álcool e outras drogas não é uma prática rotineira dos profissionais de saúde destes estabelecimentos.

\section{Discussão}

O diagnóstico dos serviços de Saúde Mental é fundamental para analisar a implantação de uma nova estratégia de atenção após a reforma psiquiátrica. Os resultados demonstraram que apesar do cumprimento de diversos critérios em cada uma das etapas estruturantes do ciclo da AF nos CAPS da região do Médio Paraopeba, são necessários esforços para a melhoria dos processos, principalmente no que diz respeito à oferta de atividades voltadas para a assistência, o cuidado e a segurança dos usuários da Saúde Mental.

Todas as instituições demonstram seguir uma das principais recomendações para promover o acesso e o uso racional de medicamentos ao apresentarem as listas dos medicamentos essenciais, sobressaindo-se em comparação com outros estudos $^{26,27}$. No entanto, o baixo número de Comissões de Farmácia e Terapêutica (CFT) atuantes se assemelha com outros estudos nacionais que reforçam não somente a deficiência no número de instâncias, mas a necessidade de aprimorar mecanismos de avaliação e monitoramento para que os trabalhos dos comitês contribuam ainda mais para a seleção de medicamentos no país ${ }^{28}$. As atividades de seleção de medicamentos internos nas unidades de saúde podem contribuir com informações direcionadas, detalhando possibilidades e limites de utilização de medicamentos pelos profissionais ${ }^{29}$. Observa-se que a falta de adesão dos profissionais pelo uso de Protocolos em serviços relativamente novos como os CAPS, é um fator que pode dificultar a padronização e o uso racional de medicamentos.

A organização da etapa de aquisição em todos os serviços é marcada por uma característica em comum relacionada à participação no consórcio de saúde da região. Os consórcios são responsáveis por proporcionar a redução de custos e desabastecimento de medicamentos ${ }^{30,31}$, assim a participação dos municípios avaliados no Consórcio Intermunicipal de Saúde da Região pode ser um dos motivos que facilitam uma melhor estruturação da etapa de aquisição, podendo influenciar inclusive a disponibilidade de diversos itens da AF como foi verificado no estudo. 
A disponibilidade dos principais medicamentos utilizados em tratamentos da Saúde Mental nos CAPS e farmácias de referência nesta região apresentaram bons resultados, alcançando valor médio acima de $90 \%$. Ainda que outros estudos tenham avaliado a disponibilidade de medicamentos em geral, e não somente aqueles destinados à Saúde Mental, os valores encontrados para este estudo estão acima da média nacional e de outras regiões ${ }^{27,32,33}$. A existência de alguns mecanismos para controle de estoques e participação do farmacêutico na etapa de programação na maioria das farmácias se opõe à falta de informação relacionada ao histórico de desabastecimento de cada um dos medicamentos, resultado já detectado em outro estudo realizado neste mesmo estado $^{32}$. Este cenário pode refletir a necessidade de informatização e treinamento adequado dos funcionários, a cerca do correto controle de estoque em cada uma das unidades de dispensação.

Quanto às condições de armazenamento dos medicamentos, considerando que mais de $3 / 4$ dos serviços ofereciam instalações adequadas, ou seja lugar limpo, amplo e arejado, observou-se uma média superior à encontrada na Avaliação Nacional da Assistência Farmacêutica ${ }^{27}$ em outras cidades e seguimentos também já avaliados neste requisito ${ }^{34}$. A área de dispensação também seguiu grande parte dos critérios recomendados. Este resultado isenta os serviços de um problema estrutural grave, porém muitas vezes revela a inexistência de padronização para a atividade, ao detectar em algumas unidades a utilização de salas pequenas, estruturas improvisadas para o fracionamento dos medicamentos ou necessidade de reformas em imóveis adaptados para alocação de alguns CAPS.

Verificou-se como fator crítico, a falta de identificação e rastreabilidade dos medicamentos dispensados. O pequeno número de CAPS que dispensam os medicamentos com a identificação completa, somado a falta de mecanismos para a rastreabilidade das informações indicam o descumprimento da legislação sanitária vigente ${ }^{22}$. $\mathrm{O}$ fracionamento dos medicamentos em condições inadequadas pode induzir erros na administração dos mesmos e reflete riscos à qualidade do cuidado e segurança dos usuários.

A atuação dos farmacêuticos nas unidades de dispensação internas aos CAPS ainda é insuficiente, visto que estes profissionais são únicos nos serviços e permanecem nelas por apenas um período do horário de funcionamento. Além da coordenação de processos organizativos no ciclo da AF, cabe ressaltar que o farmacêutico é um dos profissionais responsáveis por padronizar e recomendar o uso racional de medicamentos nos serviços de saúde.

A escassez de atividades promovidas pelos farmacêuticos nos CAPS direcionadas aos usuários ou mesmo a falta de registro de intervenções atestam a deficiência do trabalho assistencial, colocando em questionamento o impacto que a falta dessa atividade pode promover na promoção e recuperação da saúde. O contato com o usuário durante e principalmente após o período de internação é muito importante, pois há diversas barreiras relacionadas à adesão ao tratamento e dificuldade frequentes no gerenciamento das terapias, que comprometem o trabalho já desenvolvido no serviço de Saúde Mental ${ }^{13,35}$.

O farmacêutico pode exercer um papel importante na cadeia de assistência à saúde. A dispensação de medicamentos é uma oportunidade de informar e educar o usuário, fator importante para o sucesso do tratamento, além de poder identificar, reduzir ou até mesmo corrigir riscos associados à sua farmacoterapia ${ }^{13,36}$. Ressalta-se que ainda são poucas as práticas de atendimento diferenciado aos usuários da Saúde Mental e intervenções farmacêuticas tanto nas farmácias da Atenção Básica quanto nos CAPS de todo país $^{13,37,38}$. Estudos apontam que serviços farmacêuticos na Saúde Mental são extremamente relevantes em outros países e demonstram resultados positivos tanto na clínica quanto na percepção e reconhecimento dos usuários ${ }^{38}$. Assim, iniciativas para a incorporação deste tipo de atividade são necessárias para o avanço e consolidação da reorientação da Assistência Farmacêutica, além de representar uma alternativa à superação de problemas frequentes dos usuários da Saúde Mental, como a melhoria na adesão ao tratamento farmacológico ${ }^{13,14}$.

O presente estudo apresentou como uma de suas limitações a impossibilidade de calcular o indicador sobre disponibilidade contínua dos principais medicamentos para todos os CAPS, uma vez que muitos serviços não apresentaram registros e informações precisas acerca do histórico de desabastecimentos que ocorreram ao longo do último ano sobre os principais farmacos da Saúde Mental. Outra limitação é a inexistência de unidades de dispensação específicas para os CAPS em algumas cidades, dificultando a identificação de ações realizadas frente ao desconhecimento de alguns funcionários ou a falta de integração entre os setores. A coleta de alguns dados por entrevistas dirigidas está sujeita à veracidade das informações fornecidas pelos profissionais 
alocados nos serviços, visto a impossibilidade de observar todas as atividades realizadas em uma pesquisa do tipo inquérito.

O uso de medicamentos no tratamento dos transtornos mentais é parte fundamental das atividades dos CAPS e os achados deste estudo indicam aspectos capazes de influenciar o acesso e a qualidade das atividades prestadas e, principalmente, prioridades na tomada de decisão. Este estudo apresenta dados inéditos sobre as condições da AF nos CAPS, ao mesclar critérios de avaliação da AF e as especificidades dos serviços de Saúde Mental, preenche uma lacuna científica de ambas as áreas, extrapolando o conteúdo de ava- liações da Saúde Mental que não incluem questões relativas aos medicamentos e estudos sobre AF que não foram conduzidos especificamente em unidades de Saúde Mental.

Conclui-se que a identificação e a rastreabilidade dos medicamentos dispensados nos CAPS necessitam de adequação aos critérios requeridos pela legislação sanitária, bem como a consolidação de serviços farmacêuticos. Estes aspectos poderão contribuir para melhor manejo, segurança do paciente e assertividade da equipe multidisciplinar, proporcionando de fato a execução de cada uma das atividades da assistência farmacêutica e a integração entre as mesmas.

\section{Colaboradores}

SN Silva e MG Lima contribuíram na concepção, análise de dados e redação do manuscrito. 


\section{Referências}

1. Kessler RC, Aguilar-Gaxiola S, Alonso J, Chatterji S, Lee S, Ormel J, Ustün TB, Wang PS. The global burden of mental disorders: an update from the WHO World Mental Health (WMH) surveys. Epidemiol Psichiatr Soc 2009; 18(1):23-33.

2. World Health Organization. Mental health action plan 2013-2020 [Internet]. Geneva: 2013. [acessado 2016 jan 20]. Disponível em http://apps.who.int/iris/bitstre am/10665/89966/1/9789241506021_eng.pdf?ua=1

3. Whiteford HA, Degenhardt L, Rehm J, Baxter AJ, Ferrari AJ, Erskine HE, Charlson FJ, Norman RE, Flaxman AD, Johns N, Burstein R, Murray CJ, Vos T. Global burden of disease attributable to mental and substance use disorders: findings from the Global Burden of Disease Study 2010. Lancet 2013; 382(9904):1575-1586.

4. Andrade LH, Wang Y-P, Andreoni S, Silveira CM, Alexandrino-Silva C, Siu ER, Nishimura R, Anthony JC, Gattaz WF, Kessler RC, Viana MC. Mental disorders in megacities: findings from the Sao Paulo megacity mental health survey, Brazil. PloS one [periódico na Internet] 2012; 7(2):[cerca de 8 p.]. Disponível em: http:// journals.plos.org/plosone/article?id=10.1371/journal. pone. 0031879

5. Gonçalves DA, Mari JDJ, Bower P, Gask L, Dowrick C, Tófoli LF, Campos M, Portugal FB, Ballester D, Fortes S. Brazilian multicentre study of common mental disorders in primary care: rates and related social and demographic factors. Cad Saude Publica 2014; 30(3):623632.

6. Brasil. Ministério da Saúde (MS). Portaria no 336, de 19 de fevereiro de 2002. Define e estabelece diretrizes para o funcionamento dos Centros de Atenção Psicossocial. Diário Oficial da União 2002; 12 fev.

7. Nascimento ADF, Galvanese ATC. Evaluation of psychosocial healthcare services in the city of Sao Paulo, Southeastern Brazil. Rev Saude Publica 2009; 43(Supl. 1):8-15.

8. Nunes M, Jucá VJ, Valentim CPB. Ações de saúde mental no Programa Saúde da Família: confluências e dissonâncias das práticas com os princípios das reformas psiquiátrica e sanitária. Cad Saude Publica 2007; 23(10):2375-2384.

9. Brasil. Ministério da Saúde (MS). Saúde Mental em Dados - 12, ano 10, no 12. [Internet]. Brasília: outubro de 2015. [acessado 2016 jan 20]. Disponível em http:// www.saude.gov.br/bvs/saudemental

10. Brasil. Portaria GM/MS no 130 de 26 de janeiro de 2012. Redefine o Centro de Atenção Psicossocial de Álcool e outras Drogas 24 h (CAPS AD III) e os respectivos incentivos financeiros. Diário Oficial da União 2012; 27 jan.

11. Organização Mundial da Saúde (OMS). Relatório sobre a saúde no mundo 2001: Saúde mental: nova concepção, nova esperança. [Internet]. Geneva: OMS; 2001. [acessado 2016 jan 20]. Disponível em: http://www.who.int/ whr/2001/en/whr01_djmessage_po.pdf

12. Brasil. Ministerio da Saude (MS). Secretaria de Políticas de Saude. Política Nacional de Medicamentos. Brasília: MS; 2001.

13. Zanella CG, Aguiar PM, Storpirtis S. Atuação do farmacêutico na dispensação de medicamentos em Centros de Atenção Psicossocial Adulto no Município de São Paulo, SP. Cien Saude Colet 2015; 20(2):325-332.
14. Alencar TOS, Cavalcante EAB, Alencar BR. Assistência farmacêutica e saúde mental no Sistema Único de Saúde. Rev Ciênc Farm Básica e Apl 2013; 33(4):489-495.

15. Wang I, Dopheide JA, Gregerson P. Role of a Psychiatric Pharmacist in a Los Angeles "Skid-Row" safety-net clinic. J Urban Health 2011; 88(4):718-723.

16. Rubio-Valera M, Chen TF, O'Reilly CL. New roles for pharmacists in community mental health care: a narrative review. Int J Environ Res Public Health 2014; 11(10):10967-10990.

17. Oliveira MAF, Cestari TY, Pereira MO, Pinho PH, Gonçalves RMDA, Claro HG. Processos de avaliação de serviços de saúde mental: uma revisão integrativa. Saúde Debate 2014; 38(101):368-378.

18. Araújo ADLA, Pereira LRL, Ueta JM, Freitas O. Perfil da assistência farmacêutica na atenção primária do Sistema Único de Saúde. Cien Saude Colet 2008; 13(Supl.):611-617.

19. World Health Organization. WHO operational package for assessing, monitoring and evaluating country pharmaceutical situations: guide for coordinators and data collectors. [Internet]. Geneva: 2007. [acessado $2016 \mathrm{mar}$ 10]. Disponível em: http://apps.who.int/medicinedocs/ documents/s14877e/s14877e.pdf

20. Marin N, Luiza VL, Osorio-de-Castro CGS, Machado-dos-Santos S. Assistência farmacêutica para gerentes municipais. Assistência farmacêutica para gerentes municipais. Brasília: Organização Pan-Americana da Saúde; 2003.

21. Brasil. Ministério da Saude (MS). Assistência Farmacêutica na Atenção Básica: Instruções Técnicas para sua organização. Brasília: MS; 2006.

22. Brasil. Ministério da Saúde (MS). Portaria SVS/MS no 344, de 12 de maio de 1998. Aprova o Regulamento Técnico sobre substâncias e medicamentos sujeitos a controle especial. Diário Oficial da União 199831 dez.

23. Brasil. Resolução 44/2009, de 17 de agosto de 2009 . Dispõe sobre Boas Práticas Farmacêuticas para o controle sanitário do funcionamento, da dispensação e da comercialização de produtos e da prestação de serviços farmacêuticos em farmácias e drogarias e dá outras providências. Diário Oficial da União 2009; 18 ago.

24. Brasil. Ministério da Saúde (MS). Relação Nacional de Medicamentos Essenciais RENAME 2013. [Internet]. 2013. [acessado 2016 mar 10]. Disponível em: http:// portalsaude.saude.gov.br/images/pdf/2013/outubro/21/rename-anexos-versao-08-08-2013.pdf

25. Instituto Brasileiro de Geografia e Estatística (IBGE). Informações sobre os municípios brasileiros, 2014. [Internet]. 2014. [acessado 2015 jan 30]. Disponível em http:www.cidades.ibge.gov.br

26. Magarinos-Torres R, Pepe VLE, Oliveira MA, Osoriode-Castro CGS. Medicamentos essenciais e processo de seleção em práticas de gestão da Assistência Farmacêutica em estados e municípios brasileiros. Cien Saude Colet 2014; 19(9):3859-3868.

27. Jaramillo NM, Ivama AM, Barbano DBA, Santos MRC, Luiza VL, organizadores. Avaliação da assistência farmacêutica no Brasil. Brasília: Organização Pan-Americana da Saude; 2005. 
28. Santana RS, Jesus EMS, Santos DG, Lyra Júnior DP, Leite SN, Silva WB. Indicadores da seleção de medicamentos em sistemas de saúde: uma revisão integrativa. Rev Panam Salud Publica 2014; 35(3):228-234.

29. Magarinos-Torres R, Pepe VLE, Osorio-de-Castro CGC. Estruturação da assistência farmacêutica: plano de ação para a seleção de medicamentos essenciais. Cad Saude Coletiva 2013; 21(2):188-196.

30. Amaral SMS, Blatt CR. Consórcio intermunicipal para a aquisição de medicamentos: impacto no desabastecimento e no custo. Rev Saude Publica 2011; 45(4):799801.

31. Lamb L, Pontarolli DRS, Caputo DSdP, Grochocki MHC, Jaramillo NM. Consórcio Paraná Saúde: a experiência do estado do Paraná na aquisição de medicamentos da assistência farmacêutica na atenção basica. In: Osorio-de-Castro CGS, Luiza VL, Castilho SRD, Oliveira, MA, Jaramillo NM. Assistência farmacêutica: gestão e prática para profissionais da saúde. Rio de Janeiro: Editora Fiocruz; 2014. p. 363-372.

32. Guerra Júnior AA, Acúrcio FA, Gomes CAP, Miralles M, Girardi SN, Werneck GAF, Carvalho CL. Disponibilidade de medicamentos essenciais em duas regiões de Minas Gerais, Brasil. Rev Panam Salud Publica 2004; 15(3):168-175.

33. Silva Menolli PV, Ivama AM, Júnior LC. Caracterización de los servicios farmacéuticos de atención primaria del Sistema Único de Salud en Londrina, Paraná, Brasil. Rev Panam Salud Publica 2009; 25(3):254-259.

34. Oliveira MA, do Couto Esher AFS, Santos EM, Cosendey MAE, Luiza VL, Bermudez JAZ. Avaliação da assistência farmacêutica às pessoas vivendo com HIV/ AIDS no Município do Rio de Janeiro Evaluating pharmaceutical services for people living with HIV/AIDS in the city of Rio de Janeiro. Cad Saude Publica 2002; 18(5):1429-1439.

35. Schneider JF, Camatta MW, Nasi C, Adamoli AN, Kantorski, LP. Avaliação de um centro de atenção psicossocial brasileiro. Ciencia y Enfermeria 2009; XV(3):91100.

36. Oenning D, Oliveira BV, Blatt CR. Conhecimento dos pacientes sobre os medicamentos prescritos após consulta médica e dispensação Patient awareness about drugs prescribed after medical appointment and prescription. Cien Saude Colet 2011; 16(7):3277-3283.

37. Camuri D, Dimenstein M. Processos de trabalho em saúde: práticas de cuidado em saúde mental na Estratégia Saúde da Família. Saúde Soc 2010; 19(4):803-813.

38. Lucchetta RC, Mastroianni PC. Intervenções farmacêuticas na atenção à saúde mental: uma revisão. Rev Ciênc Farm Básica e Apl 2012; 32(2):165-169.

Artigo apresentado em 03/06/2016

Aprovado em 11/11/2016

Versão final apresentada em 13/11/2016 


\section{ERRATA}

p. 2025

onde se lê:

A identificação completa dos medicamentos estava presente em apenas sete serviços e todos apresentaram falhas na rastreabilidade dos que foram dispensados.

leia-se:

A identificação completa dos medicamentos estava presente em apenas sete serviços e todos apresentaram falhas na rastreabilidade dos medicamentos que foram dispensados. 